\title{
Biofeedback in headache: An overview of approaches and evidence
}

\begin{abstract}
Biofeedback-related approaches to headache therapy fall into two broad categories: general biofeedback techniques (often augmented by relaxation-based strategies) and methods linked more directly to the pathophysiology underlying headache. The use of general biofeedback-assisted relaxation techniques for headache has been evaluated extensively by expert panels and meta-analyses. Taken together, these reviews indicate that (1) various forms of biofeedback are effective for migraine and tension-type headache; (2) outcomes with biofeedback rival outcomes with medication therapy; (3) combining biofeedback with medication can enhance outcomes; and (4) despite efficacy in many patients, biofeedback fails to bring significant relief to a sizeable number of headache patients. Biofeedback methods that more directly target headache pathophysiology have focused chiefly on migraine. These headache-specific approaches include blood volume pulse biofeedback, which has considerable supportive evidence, and electroencephalographic feedback.
\end{abstract}

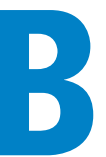
iofeedback has long been employed for helping ameliorate symptoms of recurrent headache; seminal work was performed in the late 1960s and first reported in the early 1970s. ${ }^{1,2}$ This early work focused mainly on electromyography (EMG) or muscle tension and hand temperature. Today a greater array of approaches are available, and they fall within two broad categories: (1) biofeedback-assisted relaxation and (2) specific or more specialized approaches. ${ }^{3}$

The first category employs the two types of biofeedback mentioned earlier (EMG and thermal feedback), as well as feedback on sweat gland activity, to counteract the sympathetic nervous arousal that occurs in response to stress for a host of disorders, not just headache. These types of biofeedback are commonly augmented with a variety of allied relaxation-based strategies (guided imagery, diaphragmatic or paced breathing, autogenic

Dr. Andrasik reported that he has no financial relationships that pose a potential conflict of interest with this article.

doi:10.3949/ccjm.77.s3.13 training, meditation, etc) as well as training in cognitive and behavioral stress coping. The second category takes a different approach, applying techniques that seek more directly to target the aberrant physiology underlying specific headache types. This latter category has focused chiefly on migraine headache and its variants.

This article reviews the supportive evidence for each category of biofeedback approaches to headache therapy and identifies select areas for future research attention.

\section{EVIDENCE BASE FOR GENERAL BIOFEEDBACK TECHNIQUES IN HEADACHE}

Biofeedback-assisted relaxation approaches for headache have been evaluated extensively over the past several decades. These evaluations have consisted of two basic types-comprehensive reviews by expert panels, and meta-analytic statistical analyses-as detailed below.

\section{Expert panel reviews}

A wide variety of groups have assessed biofeedback and related relaxation-based procedures by reviewing all relevant published studies according to rigorous predetermined criteria. These groups include the National Institutes of Health, the Canadian Headache Society, the American Psychological Association, the Society of Pediatric Psychology, the Association for Applied Psychophysiology and Biofeedback, and the US Headache Consortium.

The 2000 evidence review by the latter group, the US Headache Consortium, ${ }^{4}$ merits particular mention, for several reasons. First, their review was sponsored by diverse medical societies - namely, the American Academy of Family Physicians, American Academy of Neurology, American Headache Society, American College of Emergency Physicians, American College of Physicians-American Society of Internal Medicine, American Osteopathic Association, and National Headache Foundation. Second, this review panel applied objective criteria, grading the evidence quality as A, B, or C (see Table 1 for details). Third, the panelists examined a diverse array of behavioral and physical treatments (acupuncture, transcutaneous electrical nerve stimulation, occlusal adjustment, cervical manipulation, and 
TABLE 1

Treatment recommendations on behavioral and physical treatments for migraine from US Headache Consortium evidence-based guidelines ${ }^{4}$

Relaxation training, thermal biofeedback combined with relaxation training, electromyographic biofeedback, and cognitive-behavioral therapy may be considered as treatment options for prevention of migraine (Grade $A$ evidence*)

Behavioral therapy (ie, biofeedback, relaxation) may be combined with preventive drug therapy to achieve additional clinical improvement for migraine relief (Grade B evidence*)

Evidence-based recommendations are not yet possible on the use of hypnosis, acupuncture, transcutaneous electrical nerve stimulation, cervical manipulation, occlusal adjustments, or hyperbaric oxygen as preventive or acute therapy for migraine (Grade C evidence*)

*Grade A: Multiple well-designed randomized controlled trials (RCTs) revealing a consistent pattern of positive findings. Grade B: Some supportive evidence from RCTs, but not optimal support (often because RCTs were few or findings were judged to be inconsistent). Grade C: Consensus on the recommendation achieved among consortium members in the absence of acceptable RCTs.

hyperbaric oxygen) previously identified in a technical review prepared for the Agency for Health Care Policy and Research, ${ }^{5}$ a review that included detailed metaanalyses as well. Fourth, the panel's main objective was to provide scientifically sound and clinically relevant practice guidelines for use in primary care settings.

Table 1 summarizes the consortium's resulting treatment recommendations on behavioral and physical treatments for migraine. ${ }^{4}$ The consortium also prepared a list of special indicators for behavioral treatment, which are summarized in Table $2 .{ }^{4}$ Thus, strong support was garnered for thermal and EMG biofeedback for migraine, and this support is consistent with findings from many meta-analyses addressing not only migraine but also tension-type headache (see next section). The panelists noted that there was insufficient information for recommending which type of treatments to pursue for specific patients, a conclusion that holds true to the present.

\section{Meta-analytic reviews}

The other major type of evaluation applied to biofeedback for headache is more quantitative in nature, applying meta-analytical statistical analyses to available studies to determine the range and mean level of clinical effects across pooled studies. Biofeedback and related approaches to headache have been subject to an extensive number of quantitative reviews, the first being published in 1980.6 Since then, approximately 15 other quantitative reviews have compared behavioral treatments with one another, with various placebo conditions,
TABLE 2

\section{Patient characteristics for which behavioral treatments for migraine may be particularly well suited*}

\section{Preference for a nondrug approach}

Intolerance of, or medical contraindication to, drug treatment

Absent or minimal response to drug treatment

Pregnancy, plans to become pregnant, or current nursing status

History of long-term, frequent, or excessive use of analgesic or other acute medications that aggravate headache symptoms or are reducing medication effectiveness

Presence of significant life stress or lack of adequate stresscoping skills

${ }^{*}$ From US Headache Consortium evidence-based guidelines. ${ }^{4}$

or with various prophylactic medications for migraine and tension-type headaches in adults and in children and adolescents. ${ }^{7}$ The most recent meta-analysis, by Nestoriuc et al, ${ }^{8}$ focused extensively on biofeedback and will be discussed in detail here.

Nestoriuc et al identified and screened 150 clinical trials, including randomized controlled trials and quasiexperimental designs. ${ }^{8}$ Ninety-four of these trials met predefined inclusion criteria (headache diagnostic criteria specified, biofeedback evaluated as treatment alone or in combination with behavior therapy, outcome assessed using a structured headache diary, 5 or more patients per condition, and sufficient data to permit calculation of effect sizes). It was possible to include a sufficient number of studies to permit comparisons with two types of control groups: waiting list and placebo.

For migraine, biofeedback treatment yielded small to medium effects overall compared with waiting-list control and placebo, although these effects failed to reach statistical significance. For tension-type headache, biofeedback treatment yielded a medium to large effect compared with waiting-list control and a medium effect compared with placebo, both of which were statistically significant. ${ }^{8}$

The accompanying figures provide a more detailed snapshot of results from the meta-analysis by Nestoriuc et al. Figure 1 shows effect sizes in terms of headache pain for various biofeedback treatments for migraine. Figure 2A shows effect sizes for all biofeedback treatments combined for migraine, while Figure 2B shows effect sizes for EMG biofeedback alone for tension-type headache (this was the only type of biofeedback with a sufficient number of studies in tension-type headache to permit analysis). Both panels of Figure 2 show effect sizes on the four main pain outcome measures used in 


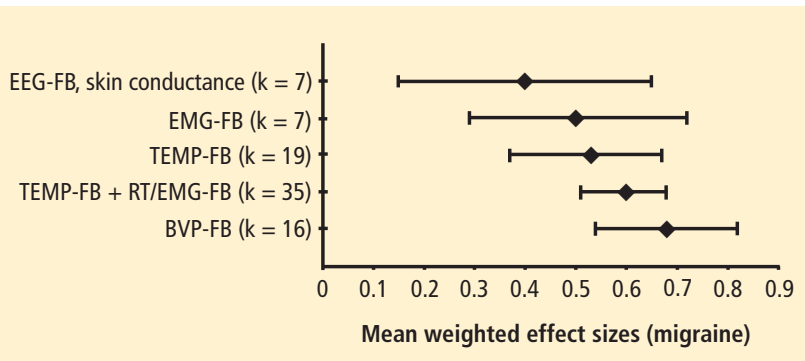

BVP-FB = blood volume pulse feedback; EEG-FB = electroencephalographic feedback; $\mathrm{EMG}-\mathrm{FB}$ = electromyographic feedback; $\mathrm{RT}$ = relaxation training; $\mathrm{TEMP}-\mathrm{FB}=$ peripheral skin temperature feedback

FIGURE 1. Mean weighted effect sizes (and 95\% confidence intervals) for migraine pain for various biofeedback methods from a meta-analysis of studies of biofeedback treatment for migraine. ${ }^{8}$ ( $k$ = number of independent effect sizes entered into the calculation) With kind permission from Springer Science+Business Media: Applied Psychophysiology and Biofeedback, "Biofeedback treatment for headache disorders: a comprehensive efficacy review," volume 33 2008, p. 131, Nestoriuc Y, Martin A, Rief W, Andrasik F, figure 1.

headache research, along with reductions in medication (considered a behavior motivated by pain). Figure 3 shows effect sizes from biofeedback on the secondary outcome measures of anxiety, depression, and selfefficacy, again for all biofeedback procedures for migraine and for EMG biofeedback alone for tension-type headache. These latter results show that biofeedback has the added advantage of favorably affecting cognitive and emotional functioning. ${ }^{8}$

Additionally, Holroyd and colleagues have conducted a number of meta-analyses and randomized controlled trials that compare behavioral and prophylactic pharmacologic treatments, as well as their combination. ${ }^{9-13}$ These reviews and studies have consistently shown that outcomes for the individual treatments are similar in magnitude and that the combination of both behavioral and pharmacologic treatment leads to even greater effects-a conclusion tentatively offered by the US Headache Consortium back in 2000. ${ }^{4}$

\section{Interim conclusions}

Consideration of the findings from individual studies and reviews discussed, plus those not singled out here, leads to the following conclusions:

1) Various forms of biofeedback are effective for migraine and tension-type headache.

2) Outcomes with these forms of biofeedback rival outcomes with medication alone.

3) Combining biofeedback with medication can enhance outcomes.

4) Outcomes from biofeedback are similar to those obtained with other behavioral approaches. Whether biofeedback has a unique advantage over other similar approaches is not known, but at least

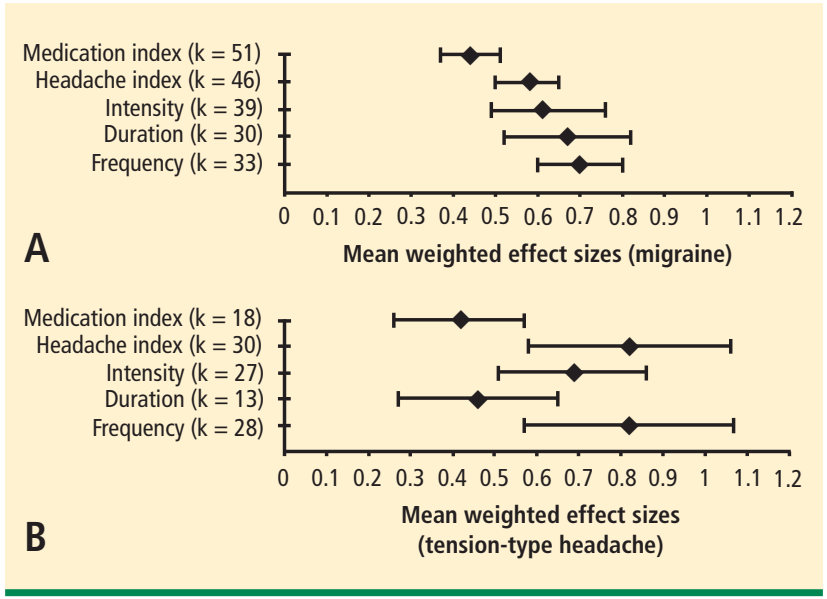

FIGURE 2. Mean weighted effect sizes (with $95 \%$ confidence intervals) for various headache outcome measures from a meta-analysis of studies of biofeedback treatment for headache. ${ }^{8}$ Results are for all biofeedback procedures combined in the treatment of migraine (A) and for electromyographic biofeedback alone in the treatment of tension-type headache (B). ( $k$ = number of independent effect sizes entered into the calculation)

With kind permission from Springer Science+Business Media: Applied Psychophysiology and Biofeedback, "Biofeedback treatment for headache disorders: a comprehensive efficacy review, " volume 33, 2008, p. 131, Nestoriuc Y, Martin A, Rief W, Andrasik F, figure 2.

one investigation suggests that biofeedback may be of particular value to a subset of patients. ${ }^{14}$

5) Although not reviewed here, the outcome effects from biofeedback seem to endure for extended periods,$^{15}$ whether booster treatments are provided or not. ${ }^{16}$

6) Although biofeedback has been shown to be effective for a number of patients, a sizeable number of patients do not achieve significant relief.

\section{Remaining questions and challenges}

Unfortunately, little attention has been devoted to identifying variables predictive of outcome. Certain headache types - chronic forms of headache (presence of pain $\geq 15$ days per month), headaches associated with the menstrual cycle, headaches accompanied by medication overuse (of ergotamine, triptans, analgesics, or opioids), posttraumatic headaches, and cluster headaches-have shown minimal response to biofeedback alone.

Headaches complicated by medication overuse are particularly difficult to treat. The first order in treatment is to have the patient withdrawn from the offending agents, which often requires a brief hospitalization, after which a more appropriate course of treatment is begun. Unfortunately, relapse is high. Mindful of this, we conducted an investigation that assigned 61 consecutive patients who had undergone a course of inpatient withdrawal to either medication alone or medication plus biofeedback-assisted relaxation training to determine if 

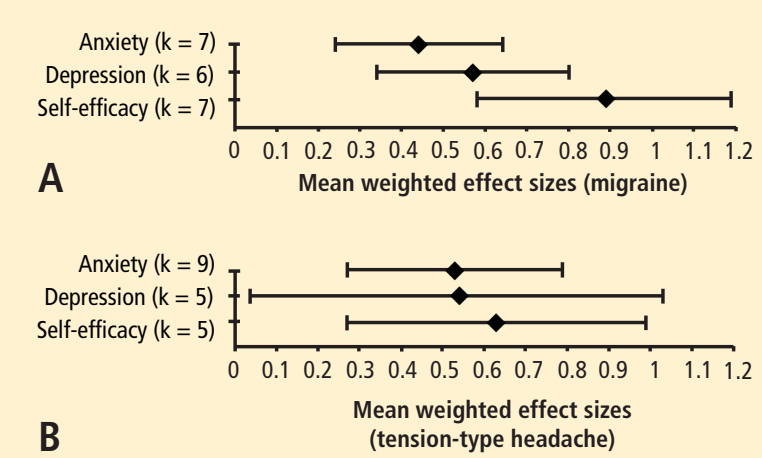

FIGURE 3. Mean weighted effect sizes (with 95\% confidence intervals) for secondary outcome measures related to cognitive and emotional function from a meta-analysis of studies of biofeedback treatment for headache. ${ }^{8}$ Results are for all biofeedback procedures combined in the treatment of migraine (A) and for electromyographic biofeedback alone in the treatment of tension-type headache (B). ( $k=$ number of independent effect sizes entered into the calculation) With kind permission from Springer Science+Business Media: Applied Psychophysiology and Biofeedback, "Biofeedback treatment for headache disorders: a comprehensive efficacy review," volume 33 , 2008, p. 131, Nestoriuc Y, Martin A, Rief W, Andrasik F, figure 2.

such training could enhance outcome. ${ }^{17}$ At 1-year follow-up evaluation, the two patient groups showed similar levels of improvement. However, at 3-year follow-up, patients receiving biofeedback showed more sustained improvements and, most importantly, had lower rates of relapse back to analgesic overuse (Figure 4). Thus, biofeedback seemed to help these patients cope more effectively over the long term. Unfortunately, we did not collect sufficient data over the intervening 2 years, so we could not determine with precision what mediated this differential outcome.

\section{EVIDENCE BASE FOR HEADACHE-SPECIFIC BIOFEEDBACK APPROACHES}

As noted above, a number of biofeedback approaches have been suggested that are tied more directly to the underlying physiology of headache.

\section{Blood volume pulse biofeedback}

One of these approaches, blood volume pulse (BVP) biofeedback, has undergone a sufficient number of trials to be included in the recent meta-analysis by Nestoriuc et $\mathrm{al}^{8}$ mentioned earlier. This approach involves monitoring blood flow in the temporal artery and providing feedback to patients to enable them to decrease or constrict blood flow. This approach, when first envisioned, ${ }^{18}$ was viewed as the nondrug counterpart to the abortive agent ergotamine. Although BVP biofeedback is not very common in clinical practice, the meta-analysis by Nestoriuc et $\mathrm{al}^{8}$ found it to produce the greatest effect size of the biofeedback methods assessed for migraine relief (Figure 1).

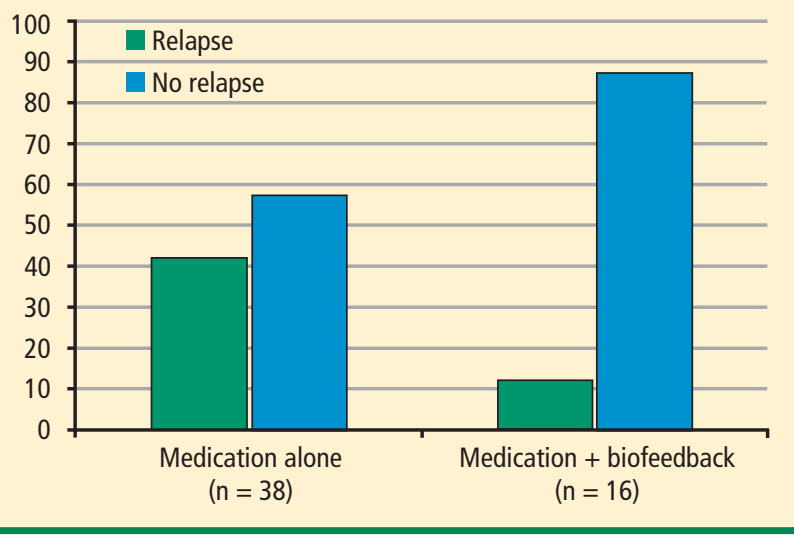

FIGURE 4. Percentage of migraine patients who relapsed to analgesic overuse at 3-year follow-up after being assigned to either medication therapy alone or medication therapy combined with biofeedback-assisted relaxation to combat initial analgesic overuse. Data are from a study by Grazzi et al. ${ }^{17}$

\section{EEG-based methods}

The next most investigated approach involves electroencephalographic (EEG) biofeedback, of which there are two types. The first derives from research investigating links between certain EEG frequency bands and the experience of pain. ${ }^{19}$ This research suggests that the experience of pain is associated with lower amplitudes of slow brain wave activity (delta, theta, and alpha) and higher amplitudes of faster brain wave activity (beta). Several uncontrolled series suggest that EEG biofeedback may be of value, but more well-controlled investigations are needed before further statements can be made.

The second line of EEG research takes a different approach, focusing on the contingent negative variation response $(\mathrm{CNV})$. The $\mathrm{CNV}$ is a slow cortical event-related potential that examines EEG activity occurring between presentation of a warning stimulus and an imperative stimulus (in this case 3 seconds later), a stimulus requiring a response by the individual. This potential is related to the level of excitability upon activation in the striatothalamocortical loop, reflecting different stages of information processing. ${ }^{20}$ Studies in child and adult migraineurs reveal that these patients have a heightened response to novel stimuli and do not habituate as readily over repeated trials as do nonmigraineur controls. ${ }^{21}$ The CNV is believed to reflect anticipation of a migraine attack because its amplitude and habituation patterns change during the headachefree interval. Abnormalities gradually increase in the days before a migraine attack, with the most pronounced changes occurring just prior to the attack. ${ }^{22}$

On the basis of these etiopathologic findings, Siniatchkin et al conducted an initial test to determine whether child migraineurs could learn, via biofeedback, to change their CNV activity and whether such learning 
would alter the subsequent course of migraine attacks. ${ }^{23}$ Ten child migraineurs without aura each received 10 sessions of CNV biofeedback. They were taught how to increase and decrease EEG negativity (as bidirectional control of a physiologic response is assumed to reflect a greater level of self-regulation). By the end of training, the children could indeed regulate their CNV activity when feedback was provided, but they were unable to do so when the feedback was removed.

The number of training sessions administered was low, as most treatment investigations using EEG biofeedback typically use 20 to 40 sessions. A greater number of sessions may have led to greater response generalization. Interestingly, baseline or tonic levels of EEG negativity changed over the course of treatment, so much so that the child migraineurs were no longer distinguishable from a matched sample of healthy controls, which suggests that the migraineurs' level of cortical excitability may have diminished. CNV biofeedback led to improvements on most measures of headache activity relative to a second group of child migraineurs who comprised a waiting-list control group..$^{23}$ These preliminary findings add to those briefly mentioned for other EEG biofeedback approaches, suggesting that further investigations are warranted.

\section{REFERENCES}

1. Budzynski TH, Stoyva JM, Adler CS. Feedback-induced muscle relaxation: application to tension headache. J Behav Ther Exp Psychiatry 1970; 1:205-211.

2. Sargent JD, Green EE, Walters ED. The use of autogenic feedback training in a pilot study of migraine and tension headaches. Headache 1972; 12:120-124.

3. Andrasik F, Lords AO. Biofeedback. In: Freeman L, ed. Mosby's Complementary \& Alternative Medicine: A Research-Based Approach. 3rd ed. St. Louis, MO: Mosby Elsevier; 2008:189-214.

4. Campbell JK, Penzien DB, Wall EM. Evidence-based guidelines for migraine headache: behavioral and physical treatments. American Academy of Neurology Web site. Available at: http://www. aan.com/professionals/practice/pdfs/g10089.pdf. Accessed March 5,2010

5. Goslin RE, Gray RN, McCrory DC, Penzien D, Rains J, Hasselblad V. Behavioral and Physical Treatments for Migraine Headache. Technical Review 2.2, February 1999. Prepared for the Agency for Health Care Policy and Research under Contract No. 290-94-2025. Available from the National Technical Information Service; NTIS Accession No. 127946.

6. Blanchard EB, Andrasik F, Ahles TA, Teders SJ, O’Keefe D. Migraine and tension headache: a meta-analytic review. Behav Ther 1980; 11:613-631.

7. Andrasik F. What does the evidence show? Efficacy of behavioural treatments for recurrent headaches in adults. Neurol Sci 2007; 28(suppl 2):S70-S77.

8. Nestoriuc Y, Martin A, Rief W, Andrasik F. Biofeedback treatment for headache disorders: a comprehensive efficacy review. Appl Psychophysiol Biofeedback 2008; 33:125-140.

9. Holroyd KA, France JL, Cordingley GE, et al. Enhancing the effectiveness of relaxation-thermal biofeedback training with propranolol hydrochloride. J Consult Clin Psychol 1995; 63:327-330.

10. Holroyd KA, O'Donnell FJ, Stensland M, Lipchik GL, Cordingley GE, Carlson BW. Management of chronic tension-type headache with tricyclic antidepressant medication, stress management therapy, and their combination: a randomized controlled trial. JAMA 2001; 285:2208-2215.

11. Holroyd KA, Penzien DB. Pharmacological vs. nonpharmacological prophylaxis of recurrent migraine headache: a meta-analytic review of clinical trials. Pain 1990; 42:1-13.

12. Holroyd KA, Penzien DB, Cordingley GE. Propranolol in the management of recurrent migraine: a meta-analytic review. Headache 1991; 3:333-340.

13. Holroyd KA, Penzien DB, Rokicki LA, Cordingley GE. Flunarizine vs. propranolol: a meta-analysis of clinical trials. Headache 1992; 32:256. Abstract 13.

14. Blanchard EB, Andrasik F, Neff DF, et al. Sequential comparisons of relaxation training and biofeedback in the treatment of three kinds of chronic headache or, the machines may be necessary some of the time. Behav Res Ther 1982; 20:469-481.

15. Blanchard EB. Psychological treatment of benign headache disorders. J Consult Clin Psychol 1992; 60:537-551.

16. Andrasik F, Blanchard EB, Neff DF, Rodichok LD. Biofeedback and relaxation training for chronic headache: a controlled comparison of booster treatments and regular contacts for long-term maintenance. J Consult Clin Psychol 1984; 52:609-615.

17. Grazzi L, Andrasik F, D'Amico D, et al. Behavioral and pharmacologic treatment of transformed migraine with analgesic overuse: outcome at 3 years. Headache 2002; 42:483-490.

18. Friar LR, Beatty J. Migraine: management by trained control of vasoconstriction. J Consult Clin Psychol 1976; 44:46-53.

19. Jensen MP, Hakimian S, Sherlin LH, Fregni F. New insights into neuromodulatory approaches for the treatment of pain. J Pain 2008; 9:193-199.

20. Elbert T. Slow cortical potentials reflect the regulation of cortical excitability. In: McCallum WC, Curry SH, eds. Slow Potential Changes in the Human Brain. Plenum, NY; 1993:235-251.

21. Kropp P, Siniatchkin M, Gerber WD. On the pathophysiology of migraine-links for "empirically based treatment" with neurofeedback. Appl Psychophysiol Biofeedback 2002; 27:203-213.

22. Siniatchkin M, Averkina N, Andrasik F, Stephani U, Gerber W-D. Neurophysiological activity before a migraine attack. Neurosci Lett 2006; 400:121-124.

23. Siniatchkin M, Hierundar A, Kropp P, Kuhnert R, Gerber WD, Stephani U. Self-regulation of slow cortical potentials in children with migraine: an exploratory study. Appl Psychophysiol Biofeedback 2000; 25:13-32.

Correspondence: Frank Andrasik, PhD, Department of Psychology, University of West Florida, 11000 University Parkway, Pensacola, FL 32514; fandrasik@uwf.edu 